\title{
Die Analytikerin und ihr Trog
}

\author{
Anna Koellreuter (Zürich)
}

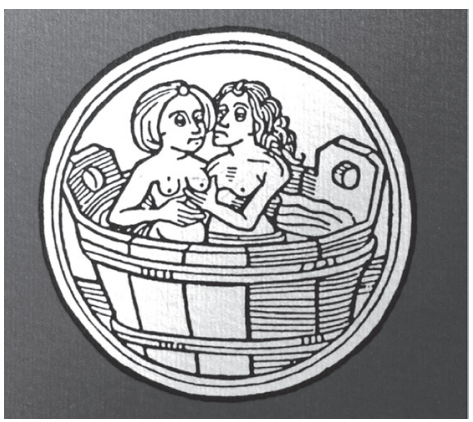

Abbildung 1: Darstellung des Titelbilds auf dem Buchumschlag von Laplanches Werk «Problématiques V. Le baquet transcendance du transfert», erschienen 1987 bei Presses Universitaires de France (PUF).

Zusammenfassung: "Le psychanalyste et son baquet ${ }^{1}-$ unter diesem Titel hielt Laplanche vor gut 40 Jahren zehn Vorlesungen zum Thema «Der Psychoanalytiker und sein Trog», womit er die psychoanalytische Situation zwischen Analytikerin und Analysandin meint. Seit seiner Publikation 1987 ist baquet (Trog) ein Begriff, der seither immer wieder in diversen französischen Wortvariationen in den Texten von Laplanche zu finden ist. Es ist ein zentraler Terminus, ohne den seine Übertragungsbegriffe schwierig zu verstehen sind. In der Übersetzung der Nouveaux fondements pour la psychanalyse, die 2011 erschienen ist, wird «baquet» auf Deutsch mit «Zuber» übersetzt. Ich bleibe beim Begriff des Troges, da dieser für mich weniger gegenständlich, sondern eher symbolisch anwendbar erscheint. In seiner Vorlesung vom 5. Dezember 1979 beschreibt er, wie er auf den Ausdruck «baquet» gestossen ist. Und zwar durch die Frage: Was spielt sich innerhalb einer Analyse ab? Gibt es ein ausserhalb der Analyse? Die Frage ist also: wie zeigen sich die Übertragungen der endlichen (innerhalb), wie jene der unendlichen (ausserhalb) Analyse? Der Trog ist ein Synonym für die hohlförmige Übertragung, auf die ich auch eingehen werde. 
Schlüsselwörter: Laplanche, Psychoanalytische Situation, Übertragung, Triebtheorie

\section{Einleitung}

Das Schwerpunktthema «Innen Zwischen Aussen» des diesjährigen Journalhefts liess mich sogleich an den Terminus baquet denken, wie auch an meine andernorts ausgeführten Überlegungen ${ }^{2}$ dazu.

«Senna perd son baquet» - das war die Schlagzeile im Figaro vom 9. Juli 2010. ${ }^{3}$ Das heisst: «Senna verliert seine Schale», womit sein Schalensitz gemeint ist, sein Behälter. Oder im übertragenen Sinne: Er verliert seinen Vertrag. Im Klartext: Er wurde aus dem Hispania Racing Team geschmissen. Bruno Senna-der Neffe des berühmten dreifachen Formel-1-Siegers Ayrton Senna, der seinerseits 1994 tödlich verunglückt ist - wurde aus seinem Sitz geschleudert, weil er nicht brachte, was von ihm erwartet wurde, nämlich die durch seinen berühmten Namen erhofften Sponsorengelder. Senna verliert seinen Schalensitz (sprich: Vertrag) und kommt nicht mehr dazu, seine Kurven zu bewältigen.

Dieses Ereignis diente mir damals als Metapher für die Situation der Analytikerin:Wird die Analytikerin aus ihrem baquet, oder Trog, katapultiert, ist sie nicht mehr in der Lage zu analysieren. Im Laplanche'schen Wortschatz ist baquet ein zentraler Begriff, der wie so manches bei ihm, auf vielfältige Weise übersetzt, gedacht und gehandhabt werden kann. Auf Deutsch übersetzt heisst baquet Becken, Bütte, Holzzuber, Zuber, Bottich, Schütte, Schale, Trog, Wanne, Behälter usw. Wie man weiss, war Laplanches Passion für den Wein genau so gross wie seine Begeisterung für die Psychoanalyse (vgl. Koellreuter, 2004; Mathey, 2004). Die halbe Woche in Pommard, die andere Hälfte in Paris - Weinproduktion und Psychoanalyse in ständiger Wechselwirkung: Der Weinbottich (baquet) kann daher als Sinnbild für die analytische Situation verstanden werden, in dem sich die von ihm beschriebene hohlförmige Übertragung abspielt (vgl. Laplanche, 1987).

Im vorliegenden Text möchte ich darlegen, wie Laplanche zu diesem Begriffund auch zu einigen anderen aussergewöhnlichen Wortkreationen - gekommen ist, wie z. B. zu den Bezeichnungen Potentialdifferenz im baquet, gefüllte und gehöhlte Übertragung oder Transzendenz der Übertragung wie weitere. Seine Texte sind leichter zu verstehen, wenn man sich mit Laplanches eigenen Wortschöpfungen befasst. Denn Neologismen, also sprachliche Neubildungen von Worten, waren für ihn ein oft verwendetes Mittel, um einen Begriff möglichst genau zu beschreiben (vgl. Laplanche, 1996). Zusammen mit seinen Kollegen kreierte er bei der 
Freud-Übersetzung «eine bestimmte Anzahl von Neologismen» (vgl. Bourguignon et al., 1989). ${ }^{4}$ Laplanche wollte dennoch kein Fanatiker des Neologismus sein (vgl. Laplanche, 1996), auch wenn dies für die Leserin oder den Leser oft so scheint. Er erklärt:

Ein Neologismus in der Freud-Übersetzung ist ebenso eine wirkliche Wortschöpfung (und man wird gewahr, dass reine Neuschöpfungen sehr selten sind) wie auch, meistens, die von mir so genannte «neologisierende Verwendungsweise»: die Wiederbelebung alter, außer Gebrauch gekommener Wörter oder einer aufgegebenen Verwendungsweise eines noch verwendeten Wortes. (Laplanche, 1996, S. 61)

Seine Wortkreationen haben ihren Ursprung in der Übersetzung des Freudschen Gesamtwerkes, sind aber sicherlich auch eine Konsequenz seiner Analyse bei Jaques Lacan, welcher seit den 1950er Jahren seinerseits unendlich viele neue Wortschöpfungen in seinen Seminaren kreierte. ${ }^{5}$ Laplanche (1996) versteht die Übersetzung als

unausweichliches analytisches Modell, sobald es um die Bildung des Seelenapparates geht, und um die Verdrängung, aber auch die Deutung, die Sublimierung etc. Schließlich - mein «nolens volens» führt mich dahin - Übersetzung als Modell des Triebs und als Trieb, der mich leitet. (S. 46)

Dem Trieb zu übersetzen und der Lust zu übersetzen begegnen wir in Laplanches Texten auf Schritt und Tritt. Dies ist das Faszinierende an seinem Werk, welches gleichzeitig auch auf die kulturellen Unterschiede zwischen der frankophonen und germanophonen analytischen Denkweise hinweist.

\section{Der Trog}

Ich komme zurück zum baquet, dem Trog. Laplanche hat über «Die psychoanalytische Situation» sowie zu «Der Psychoanalytiker und sein Trog» diverse Texte verfasst. Die zwei Titel verschränken sich, indem er an verschiedenen Stellen «Die psychoanalytische Situation - der Psychoanalytiker und sein Trog» mit einem Bindestrich zusammenführt. Denn wenn die psychoanalytische Situation selbst Übertragung ist, dann ist logischerweise die Analytikerin oder der Analytiker und 
deren Trog wesentlich mitbeteiligt, damit die Übertragung überhaupt in Gang kommt.

\section{Metaphern zum Trog}

In seiner Vorlesung vom 27. November 1979 beschreibt Laplanche (1987, S. 30), wie er auf den Ausdruck baquet gestossen ist. Was spielt sich innerhalb der Analyse ab? Was ausserhalb der Analyse? Gibt es ein ausserhalb der Analyse? Dies sei eine Frage, welche Analytiker sich stellen müssten. Gewisse Analytiker würden sich darüber hinwegsetzen, indem sie etwas zu eilig sagten: «Alles analysiert sich» («tout s'analyse», Laplanche, 1987, S. 30). Um diese Grenzen zwischen einem Innen und einem Aussen zu symbolisieren, gelangt er zum Bild des baquet, des Trogs. Seine Assoziationen führten ihn zu Anton Mesmer (vgl. Laplanche, 1987, S.30), der behauptete, dass eine ungünstige magnetische Verteilung im Körper allerlei Krankheiten bewirke und er das Gleichgewicht dieses Magnetismus wieder herstellen könne, indem er mit seinem Magneten über den Körper der Patienten streiche. Er führte hypnotische Sitzungen durch, welche die Leute mittels animalischem Magnetismus ${ }^{6}$ von ihren Krankheiten heilen sollten. Bald wurde er als Scharlatan verschrien und zog von Wien nach Paris, wo er eine Praxis eröffnete und in Kürze grossen Erfolg zeitigte, der in Einzelsitzungen nicht mehr zu bewältigen war. Der Andrang wurde so gross, dass er Holzzuber mit Wasser, Eisenspänen und Glassplittern füllte, um den Magnetismus von ihm auf diese zu übertragen und so einer grösseren Anzahl von Menschen zuzuführen. ${ }^{7}$

Man könne, meint Laplanche in seiner Assoziationskette, einerseits auch an eine Art Batterie denken, genau gesagt an einen Behälter, in welchem sich verschiedene Elemente schichten, die einen Spannungsunterschied, eine Potentialdifferenz, hervorrufen würden (vgl. Laplanche, 1987, S. 30). Andererseits, wenn man sich auf den «Beeinflussungsapparat» von Victor Tausk beziehe, könnte man meinen, dass es sich bei diesem um einen lebenden Körper handle. ${ }^{8}$ Freilich wäre dies eine Art phantasmatischer Körper. Worauf es bei diesem Bild eines Körpers jedoch ankommt, ist die Idee, dieses gegenständlich darzustellen, nämlich: um eine Potentialdifferenz herzustellen ist ein Gefäss unverzichtbar. Gemäss Laplanche liegen das hydraulische Modell - jenes eines baquet, welches die angestaute Flüssigkeit, die mit Druck entweichen will, zurückhält - und das Modell einer elektromagnetischen Batterie sehr nahe beieinander, einerseits für die Physiker, aber in gewisser Weise auch für die Analytiker (vgl. Laplanche, 1987, S.31). Mit diesen Gedanken zielt er in Richtung Triebdynamik in der Übertragungssituation, die sich im baquet der Analytikerin abspielt. 
Laplanches Ausführungen zum baquet sind gespickt mit metaphorischen Bedeutungen. So war er frappiert vom Ausdruck "to kick the bucket", was dem französischen Ausdruck «casser sa pipe» entspricht. "To kick the bucket” ist ein englisches Idiom und heisst: «sterben, verrecken, den Löffel abgeben» gleich seinem französischen Pendant. Er übersetzt den englischen Ausdruck jedoch wörtlich und kommt zu: «donner un coup de pied et renverser le baquet» - also «den Trog mit einem Fusstritt umkippen $»^{9}$ (Laplanche, 1987, S. 31). Deshalb sei der englische Ausdruck vielsagender als der französische. Das Bild von «casser sa pipe» stammt aus der napoleonischen Zeit, als in der Chirurgie eine Anästhesie nicht möglich war. Man steckte dem Patienten eine Pfeife zwischen die Zähne, worauf er beissen konnte, um die Schmerzensschreie zu unterdrücken. Wenn er unter dem Messer verstarb, sagte man «il a cassé sa pipe», auf deutsch «er hat den Löffel abgegeben». Eine komplexe anekdotische Geschichte, wie Laplanche bemerkt, und kehrt wieder zu seiner Übersetzung des französischen Ausdruckes « renverser le baquet» zurück, was bedeutet: der Trog wird umgeworfen, ausgeschüttet, und bewirkt in der Folge das plötzliche und auch definitive Abfallen der Potentialdifferenz.

\section{Von Potentialdifferenz, beziehungsweise Potentialunterschied spricht man dann, wenn zwei oder mehrere Objekte zueinander unterschiedliche Potentiale besitzen. Eine Potentialdifferenz ist also ein körperunabhängiges Maß für die Stärke eines Kraftfeldes und beschreibt das Arbeitsvermögen eines Objektes in diesem. ${ }^{10}$ (Laplance, 1987, S. 30, Hervorh. d.A.)}

Bezogen auf die analytische Situation im Trog würde ich vom triebhaften oder konflikthaften Innenraum sprechen. Die zwei Objekte (Analysandin und Analytikerin) besitzen unterschiedliche Potentiale: die eine will etwas, die andere versagt es ihr. Wenn dem nicht so wäre, wenn also der Inhalt des Troges verschüttet ist, dann sind externes und internes Niveau ausgeglichen - die Abgrenzung des baquet würde keine Bedeutung mehr haben. Es geschähe nichts mehr, pointiert gesagt: das Triebleben würde stillgelegt.

Ausgangspunkt für Laplanches baquet-Begriff ist jedoch nochmals ein anderer. Er stützt sich auf die Freudschen Modelle, welche sich oft auf das Bild einer Umwallung (frz.: «enceinte») beziehen: also ein Innenraum, der von einem Aussenraum eingegrenzt wird.

In «Jenseits des Lustprinzips» stellt Freud (1920g) sich den lebenden Organismus «in seiner größtmöglichen Vereinfachung als undifferenziertes Bläs- 
chen reizbarer Substanz» (S. 26) vor. Dieses Bläschen mit seiner reizaufnehmenden Aussenschicht «schwebt inmitten einer mit den stärksten Energien geladenen Außenwelt und würde von den Reizwirkungen derselben erschlagen werden, wenn es nicht mit einem Reizschutz versehen wäre» (Freud, 1920g, S. 26). Es ist also von einer Schutzhülle umgeben, die den Innenraum abschirmt. Laplanche meint, dass es eben dieser abgeschlossene Raum sei, welcher eine Potentialdifferenz zwischen innen und aussen etabliere, und so dem Sexuellen, dem Triebhaften, Raum gebe. In anderen Worten: Das Triebhafte zwischen Analytikerin und Analysandin kann nur in diesem geschützten Innenraum, dessen Sinnbild der Trog ist, stattfinden.

\section{Die Potentialdifferenz im Trog}

Beim baquet wird also immer die Potentialdifferenz zwischen innen und aussen angesprochen, wozu Laplanche (1987) zu diesem Denkmodell drei Charakteristika anführt:

Das erste Merkmal dieses Denkmodells ist, nach dem eben gesagten folglich, dass die Differenz zwischen innen-außen unerlässlich ist und dass sie sich energetisch definiert. Es gibt keine Umhüllung ohne Energie, die sie aufrecht erhält. Man denke an einen mit Luft gefüllten Fußball: wenn er nicht aufgeblasen ist, d. h. wenn es keinen Unterschied zwischen dem Innendruck und dem Aussendruck gibt, dann verschwindet gleichzeitig die Idee der Umhüllung. Denn die Umhüllung - als Abgrenzung des Innenraums nach außenerzeugt Spannung. Und umgekehrt ist-ohne das Vorhandensein einer Umhüllung - keine Energie vorhanden, sprich Kapazität, um eine Arbeit zwischen dem Außen und dem Innen zu produzieren. Der Fußball bleibt schlapp und unbrauchbar. Energie ist niemals anders erfassbar als in Gestalt einer Umhüllung. (S. 32 f., Hervorh. d. A.)

Ein zweites Merkmal dieses Freudschen Modells, welches Laplanche umfunktioniert, hat die Aufrechterhaltung der Konstanz auf seinem Niveau als Ziel (vgl. Laplanche, 1987, S. 33). Dies nicht einfach im Sinne einer Batterie, sondern als Batterie mit Aufladesystem oder genauer: mit Selbstregulation. Wenn das Niveau sinkt, sei das System aufzufüllen; wenn aber zu viele Energien hineinströmen, dann seien diese zu entleeren. 
Schliesslich als drittes Merkmal, erwähnt Laplanche,

verteidigt sich [...] dieses System baquet gegen Aggressionen, welche insbesondere als Risse oder Einbruchstellen den Umhüllungen zugefügt wurden. Das heißt: in dem Moment, in welchem sich ein Riss in der Wand des baquet zeigt, können sich mehr oder weniger katastrophale Modifikationen des Niveaus ereignen. (Laplanche, 1987 S. 33, Hervor. d.A.)

Als Beispiel eines Risses könnte die deplazierte Bemerkung der Analytikerin lauten: «Ich bin nicht Ihre Mutter» (Laplanche, 1987 S. 33). Dann seien interne Kräfte notwendig, die sich den externen Kräften entgegenstellen müssten, um die Einbruchstellen abzudichten. Hier drängt sich das Wort «défense», also Gegenwehr auf, auf welchem Laplanche besteht, und nicht «résistance» (Widerstand). Denn es ist eine Schutzabwehr ökonomischer und dynamischer Art, bei der es darum geht, den Kräften Gegenkräfte, dem Druck Gegendruck, entgegenzustellen (vgl. Laplanche, 1987 S.33).

\section{Der Trog, die Analytikerin und die analytische Situation}

Welche Bedeutung hat der Trog in der psychoanalytischen Situation? Wie kommt die Analytikerin zu ihrem Trog? Und vor allem: wie bleibt sie drinnen?

Als Erstes geht es um die Etablierung der Kur, der psychoanalytischen Situation - im Sinne des contrat social von Rousseau, wie Laplanche beifügt welche nicht willkürlich sei und immer wieder neu hergestellt werden müsse. Es handelt sich hier um die fixen Vereinbarungen, die bis zum letzten Moment der Analyse eingehalten werden, d. h. «es gibt keinen Übergang zwischen der Analyse und der Außer-Analyse» (Laplanche, 2011, S. 189). Die Analyse spielt sich quasi intramural ab, dazu brauche es nicht nur einen Fauteuil und eine Couch, sondern vor allem auch eine geschlossene Türe! (Laplanche, 1987, S. 211; Hervorh. d. A.).

Zweitens ist gemäss Laplanche diese Errichtung der Kur ein reiner Triebort oder ein Ort des Sexuellen. Denn innerhalb des Troges geschieht alles im Zusammenhang mit Sexualität, mit Liebe und mit Hass. Die auf Anpassung abzielenden Interessen ( «intérêts adaptatifs») werden zwar nicht ausgeschlossen, aber tangentialisiert. Das heisst: sie werden beiseitegeschoben, bis sie das, was im Trog geschieht, nur noch berühren oder streifen. Mit derVerweigerung der Analytikerin, sich auf die Anpassungsebene zu begeben, z. B. indem sie Ratschläge erteilt, bleiben die Anpassungstendenzen tangential und was sich im Trog abspielt, nämlich das 
Sexuelle, Triebhafte, wird nicht gefährdet. Zusätzlich hilft auch der erste erwähnte Punkt - die fixen Vereinbarungen - dabei, die Anpassungsneigungen auf eine tangentiale Ebene zu bringen.

Hier wäre zu erwähnen, dass das Feld der Psychoanalyse durch seine wichtigste Koordinate definiert wird: nämlich weder durch die Sprache noch durch das Phantasmatische, sondern durch das Sexuelle (vgl. Laplanche, 1987, S. 148), das allerdings auch phantasmatisch sein kann. Dies auf dreifache Weise: Erstens finden wir sozusagen in allem, was in der Analyse besprochen wird, eine sexuelle Bedeutung. Zweitens muss die erwachsene Sexualität immer wieder auf die infantile Sexualität hin bezogen sein und drittens muss die Sexualität hier in ihrer erweiterten Bedeutung angenommen werden, was von den Analytikerinnen selbst oft vergessen wird. Denn die Sexualität ist nicht zu verwechseln mit der Genitalität. Die von Freud entdeckten Sexualitäten werden klassischerweise anal, oral, phallisch, urethral usw. benannt. Die Vorstellung der Sexualität definiert sich ausserhalb des Verhältnisses zu den genitalen Organen und dadurch auch des Verhältnisses zur Konzeption (vgl. Laplanche, 1987, S. 148). Der Begriff der Sexualität wurde von Laplanche als das Sexuelle, das Sexuale, das Unbewusste bezeichnet.

Der dritte Punkt beinhaltet die analytische Situation als Wiederherstellung des Ortes der Urverführung. Sie konstituiert die ursprüngliche Beziehung zum Rätsel und seinem Träger, nämlich jenem, «dem Wissen unterstellt wird». Hier zeigt sich das Wesentliche der analytischen Ethik im Zusammenhang mit der Gegenübertragung, die Laplanche suspekt erscheint. Denn es geht nicht darum, die Gegenübertragung zu beherrschen, zu gebrauchen, sich darin verstricken oder affektiv berühren zu lassen. Sondern wichtig ist, dass die Analytikerin, als jener der Wissen unterstellt wird ${ }^{11}$, dieses Wissen verweigert - und zwar der Analysandin gegenüber gleichermassen wie sich selbst. Ich möchte betonen: vor allem sich selbst! Denn verweigert sie das Wissen nicht, verwickelt sie sich automatisch in die Übertragung der Analysandin. Dies ist die zweite Verweigerung, nebst der Verweigerung, sich auf die Anpassungsebene einzulassen. Der eigentliche Motor für die Analysandinnen wissen zu wollen ist, wie beim kleinen Kind, die Folge der Zurückweisung desWissens und $z u$ wissen der Analytikerin (vgl. Laplanche, 1994).

Und schliesslich ist die Situation - der Trog - ein Ort der Haltung oder Fassung, im Französischen "contenance», der dauernd gewartet oder unterhalten werden müsse. Keinesfalls sei dies gleichzusetzen mit Winnicotts oder Bions containment! ${ }^{12}$ Laplanche vergleicht das baquet mit einer Art Zyklotron, in welchem die Teilchen mit beträchtlicher Geschwindigkeit beschleunigt werden. Ohne Umwallung oder Umgrenzung würde der Zyklotron zur Wasserstoffbombe wer- 
den (vgl. Laplanche, 1994, S. 155)! Ich verstehe den Ausdruck contenance als «die Fassung (oder auch die Selbstbeherrschung) bewahren»- egal was kommen mag. Die Gelassenheit in schwierigen analytischen Situationen aufrechterhalten oder die Contenance bewahren - wie man, mit Knigge (2006 [1796]), auch sagen kann.

Im Folgenden nochmals die vier Kriterien, welche die analytische Situation beschreiben:

1. Die festen Vereinbarungen, an welche sich beide Seiten zu halten haben.

2. Die Aufrechterhaltung des Sexuellen, Triebhaften in der Kur.

3. Die Weigerung der Analytikerin zu wissen und schliesslich:

4. Die Fassung bewahren, immer wieder neu.

Die analytische Situation besteht aus der «Psychoanalytikerin und ihrem Trog» und, wir wissen, dass die analytische Situation selbst Übertragung ist. Für Ida Macalpine und Daniel Lagache, auf welche Laplanche sich an diversen Stellen immer wieder bezieht, wird die Übertragung durch die Analyse «hergestellt» (Laplanche, 1987, S. 23, Hervorh. i. O.). Laplanche geht jedoch noch einen Schritt weiter. Für ihn ist die analytische Situation eine Neuetablierung der Ursituation (Laplanche, 1987, S. 29), und dadurch mit einer anderen Sichtweise verbunden. ${ }^{13}$

Es folgt ein kurzer Exkurs zur Ursituation, Urverführung und rätselhaften Botschaft, welche in der Übertragung eine Rolle spielen.

\section{Die Etablierung der Ursituation im baquet}

Mit Ursituation oder Urverführung ist die anthropologische Grundsituation gemeint, jene, bei der jedes Kind von rätselhaften Botschaften der Eltern (oder Erwachsenen) überflutet wird. Rätselhaft nicht im Sinne von mysteriös, sondern vom zweiseitigen Erleben, wie Laplanche (1998) erklärt:

[D]er Erwachsene hat ein Unbewusstes, das insbesondere durch die Beziehung zu diesem Winzling wachgerüttelt wird, der er selbst gewesen ist. Botschaften, die meistens nicht verbal sind: Pflege, Mimik, Gebärden, manchmal aber auch verbal. Botschaften, die ich kompromittiert nenne, weil sie nicht nur ihren manifesten Sinn übermitteln, sondern auch ihre Kompromittierung durch die unbewussten Signifikanten: "Kompromittiert», genau wie dies Freud für die Fehlhandlungen, das Versprechen, das Verschreiben, etc. gezeigt hat. Rätselhaft sind sie für den Aufnehmenden nur, weil sie auch rätselhaft für den Sender sind. (S. 615) 
Also rätselhaft und nicht-dekodierbar für beide Seiten. Fürsorge und Bindungsverhalten der Mutter sind in eine libidinöse Hülle eingebettet und ganz und gar durchtränkt von ihrer Erotik und Liebe, aber auch von ihren unbewussten Konflikten und Frustrationen. Beides wird vom Kind aufgenommen. Das Einbrechen des sexuell Anderen, also des Diskurses der Eltern mit ihren eigenen Verdrängungen und Konflikten in die organische Lebensordnung, ist für das Neugeborene potenziell traumatisch. Ohne dass es dafür ausgerüstet wäre, versucht das kleine Kind die rätselhaften Botschaften des Anderen oder der Anderen zu übersetzen, sich anzueignen, zu symbolisieren. Es gibt da etwas, das zwischen den Worten der Eltern liegt, d.h. es gilt zwischen den Zeilen zu lesen, wo das Begehren $\mathrm{zu}$ vermuten ist und für das es letztlich Worte zu finden gilt (vgl. Fink, 2005), die jedoch so schwer zu finden sind. Genau das begründet die wesensmässige Konflikthaftigkeit des Menschen und seiner Konflikte, mit denen wir es täglich in der psychoanalytischen Arbeit zu tun haben, und die sich im Trog abspielen.

\section{Die Provokation der Übertragung durch die Analytikerin}

Was macht nun die psychoanalytische Situation aus, fragt Laplanche (1996) und zählt die drei Funktionen der Analytikerin resp. des Analytikers auf:

Man kann es formulieren, es noch einmal formulieren: Ich habe mich, mit dem Bild des Trogs, lange daran versucht. Hier werde ich drei Dimensionen vorschlagen, drei Funktionen des Analytikers und dessen, was er bewirkt: der Analytiker als Garant der Konstanz; der Analytiker als Steuermann der Methode und Begleiter des Primärvorganges; der Analytiker als Hüter des Rätsels und Provokateur der Übertragung. (S. 191)

Ohne die beiden ersten Funktionen ist Analysieren nicht möglich. Analysieren heisst auflösen aller psychischen, ich-haften, ideologischen und symptomatischen Bindungen. Alles wird aus seinen Bindungen gelöst, alles Funktionale wird ausgeschlossen, oder anders gesagt: entbunden. Dazu eine Illustration von Pierre Passett (2007):

Wenn die Analysandin zu spät in die Stunde kommt und erklärt, das Tram sei mit einem Auto kollidiert und nicht weitergefahren, sie habe zu Fuss kommen müssen und darum sei sie zu spät, dann bindet sie das Zuspätkommen und ihre Mitteilung davon 
in einen vernünftigen Zusammenhang, den wir ausserhalb der Analyse akzeptieren würden. Das Ereignis wäre gleichzeitig erklärt und erledigt. Nicht so in der Analyse. Wir sind berechtigt, diese Mitteilung aus ihrem Zusammenhang zu lösen, zu entbinden, als ob sie damit nichts zu tun hätte und sie mit etwas völlig anderem zu verbinden, z. B. mit dem Ende der letzten Stunde. "Als Sie letztes Mal hier weggingen, waren Sie wütend auf mich», könnte der Analytiker z. B. sagen. Wenn das der Chef zur Mitarbeiterin sagt, die aus dem gleichen Grund zu spät kommt, liegt er völlig daneben und die Angestellte wird das so empfinden. Nicht so die Analysandin. Sie wird, wenn sie die Spielregeln einhält, auf diese Deutung mit Einfällen reagieren und diese Einfälle werden ganz woanders hin führen als zur Tramkollision, aber möglicherweise auch zum Ende der letzten Stunde. Es werden Elemente zum Vorschein kommen, die sonst verborgen geblieben wären und die werden sich vielleicht ihrerseits in andere Zusammenhänge einordnen. Das ist allerdings nicht der Fall, wenn der Analytiker etwa sagen würde: «Ihr Zuspätkommen hat letztlich mit der Tramkollision nichts zu tun, Sie kommen viel mehr zu spät, weil Sie mich bestrafen wollen, nachdem Sie in der letzten Stunde über meine Deutung wütend geworden sind», oder noch schlimmer: «Sie wollen mich kastrieren, indem Sie meine Stunde verkürzen.

In diesem Fall analysiert der Analytiker nicht, er öffnet nichts, sondern er verschliesst etwas, indem er dem be-wussten Sinn der Mitteilung der Analysandin einen zweiten anderen, von ihm ge-wussten Sinn entgegenstellt. Das wäre im Sinne Laplanches keine Deutung, sondern Hermeneutik, wahrscheinlich schlechte Hermeneutik. Laplanche (1998) sagt dazu

Hände weg von der Hermeneutik, von unserer Hermeneutik in der Kur! Dabei handelt es sich um eine regulative Maxime, die nur asymptotisch befolgt werden kann; eine andere Formulierung wäre die von der «Versagung des Wissens» seitens des Analytikers. (S. 617, Hervorh. i. O.)

Laplanches Text «Von der Übertragung und ihrer Provokation durch den Analytiker» (1996) trug ursprünglich den Titel «Die gewöhnliche und die ausser- 
gewöhnliche Übertragung». Die Gedankengänge dieser Arbeit verdeutlichen die Triebdynamik im Analyseprozess. Er unterscheidet die Analyse an der Übertragung von der Analyse in der Übertragung. Freud sah die Übertragung als Übertragung von ungelösten Konflikten, die zur Neurose führen. Folglich arbeitete er an der Übertragung (s. auch bei Freud, 1937d). Demgegenüber sieht Laplanche in der Übertragung die grössere Relevanz. Denn in der Übertragung spielt sich das Triebhafte, Sexuelle ab. Es existieren dafür keine Worte, weil das Fremde, das Sexuelle, nicht zu benennen ist. Es geht um das Bewusstsein und damit um das Aushalten des eigenen Fremden, Triebhaften. Wird die innere Fremdheit abgespalten, d.h. das Triebhafte verdrängt, dann beschränkt sich die Analyse auf die Arbeit an der Übertragung, was identisch mit dem sekundärprozesshaften - und nicht mit dem triebhaften - Geschehen wäre. Stagnationen im Analyseprozess entstehen dort, wo die Primärprozesse unbewusst bleiben oder mit Laplanche gesagt: wo die Beziehung zum Unbekannten nicht ausgehalten werde, weil es so schwer fassbar sei und deshalb ängstige.

Es gibt also für die Analytikerin die Unterscheidung, an der Übertragung versus in der Übertragung zu arbeiten, was uns zu den Laplanche'schen Begriffen der gefüllten und gehöhlten Übertragung führt.

\section{Die gehöhlte und die gefüllte Übertragung}

In seinem Kapitel «Le transfert» (1994) - die Übertragung - beschreibt Laplanche die gefüllte und die gehöhlte oder hohlförmige Übertragung, welche nebeneinander stattfänden. Die gefüllte Übertragung ist diejenige, die von Freud als typische Übertragungssituation beschrieben wird. Sie besteht in der Wiederholung archaischer Situationen, die als Füllsel bei der Analytikerin deponiert werden. Die gehöhlte Übertragung, welche in ständiger Wechselwirkung mit der gefüllten Übertragung steht, bedeutet, dass das Hohle der Analysandin in der Höhlung der Analytikerin untergebracht wird (vgl. Laplanche, 1996). Die Höhlung der Analytikerin ist «die innere wohlwollende Neutralität unserem eigenen Rätsel gegenüber» (Laplanche, 1996, S. 194). Das heisst, letztlich sind wir Analytiker uns fremd und dieser unserer eigenen Fremdheit gegenüber offen. Bringt die Analysandin ihr Hohles in die Höhlung der Analytikerin, bedeutet dies: Sie bringt in der Höhlung der Analytikerin ihre eigene Höhlung unter, d.h. das Rätsel ihrer ursprünglichen infantilen Situation wird bei der Analytikerin deponiert, ohne gedeutet zu werden. Eben das ist die hohlförmige Übertragung - in welcher sich das Triebhafte und Sexuelle abspielt. Auch hier erinnert die Metapher des Troges an eine Höhle, einen geschlossenen Raum. 
In der feministisch-psychoanalytischen Literatur und Praxis ist oft vom symbolischen Raum der Analytikerin die Rede, welchen sie der Analysandin zur Verfügung zu stellen hat (bspw. N. Chodorow, J. Benjamin, C. Gilligan, C. Olivier). Ob sich die Bedeutungen von Laplanches Höhlungmit jener der feministisch-psychoanalytischen Literatur decken, ist nicht ganz klar. Würde Laplanches Höhlung als sexuell bezeichnet und der symbolische Raum als narzisstisch, dann wären die Bedeutungsübergänge fliessend, $d$. h. beide triebhaft besetzt. Wird der symbolische Raum jedoch als Raum zur Restaurierung des beschädigten Selbst verstanden, also mehr im Sinne der Container-Funktion, dann stehen sich die beiden Auffassungen diametral gegenüber (vgl. Koellreuter, 2001).

Die Analysandin sollte sich im Trog einquartieren können - um sich dort zu öffnen und sich zu analysieren: «Analyse, das heißt die Lösung (...). Es gibt keine Auflösung der Übertragung als solcher, es gibt eine Lösung oder Auflösung der gefüllten Übertragung in die hohlförmige Übertragung» (Laplanche, 1996, S. 195). So macht die hohlförmige Übertragung die Analyse der Füllsel möglich, ohne dass sie aufgelöst werden muss - was auch gar nicht möglich wäre.

Übertragungen verlaufen zyklisch, wiederholen sich damit regelmässig. Sie sind nie beendet. In der hohlförmigen Übertragung enthalten die beiden Höhlungen, der Analytikerin und der Analysandin, die rätselhaften Botschaften der jeweiligen Kindheit, welche dem Kind vom Erwachsenen übermittelt wurden. Es sind eben diese sexuellen Rätsel, die der Erwachsene seinerseits nicht zu dekodieren imstande ist.

Schliesslich stellt sich die Frage, was mit der hohlförmigen Übertragung am Ende einer Analyse geschieht. Dazu sagt Laplanche, dass die Analysandin ihre «Übertragungsmöglichkeiten» welche sie in der Analyse gewonnen habe, an Orten innerhalb und ausserhalb der Analyse anwenden und so ihr individuelles Schicksal bestimmen könne. Denn:

Die hohlförmige Übertragung ist nicht das Resultat einer Entwicklung oder eines Prozesses. Sie ist nicht nach den Kriterien von Normalität und Abnormalität messbar. Sie ist die Grundlage der Übertragung, ihre irreduzible Dimension von Alterität. (Laplanche, 1996, S. 200, Hervorh. d. A.)

Was heisst: die unlösbare Dimension der eigenen Fremdheit - also niemals lösbar! Oder anders formuliert: Es handelt sich um die nicht änderbare Tatsache, niemals Herrin im eigenen Hause zu sein. Dies impliziert, dass das Triebhafte, das 
Fremde in Wechselwirkung der Übertragungen von Analytikerin und Analysandin immer wieder von Neuem zum Vorschein kommen muss. Geschieht dies nicht, kommt es zur Spaltung. In der Abspaltung der eigenen inneren Fremdheit (=Alterität) liegt die Angst vor der äusseren Alterität, d.h. vor dem Fremden im Anderen begründet. Bekannt sind uns die Abwehrmechanismen des Fremden, des Sexuellen, in Form von Überwindung der Differenz oder Angleichung an die Anderen (vgl. K. Flaake, 1995, u.a.m.). Oder der umgekehrte Mechanismus kommt in Gang: die Anderen werden ausgesondert oder auch vernichtet. Volkmar Sigusch (1984) formuliert es dahingehend, dass die abgespaltene Angst vor dem eigenen Fremden nicht mehr spürbar sei, damit einhergehend aber auch nicht mehr die sexuelle Lust.

\section{Transzendenz der Übertragung}

Noch einige Bemerkungen zur «Transzendenz der Übertragung» (transcendance $d u$ transfert), was für Laplanche vor 40 Jahren nicht gleichbedeutend mit der Begrifflichkeit von «Übertragung der Übertragung» war, wie ich das oben beschrieben habe. In den Nouveaux fondements (1994) wird zwar transcendance als Transzendenz übersetzt, aber es fehlen Laplanches Gedankengänge dazu, was in einer Fussnote hätte erwähnt werden können. Denn es war eine andere Bedeutung von ihm gedacht. ${ }^{14}$ Weshalb er sich von dieser abgewendet hat, ist nicht so klar. ${ }^{15}$ Denn die Transzendenz kommt in den Nouveaux fondements, sowie in seinen späteren Texten nirgends mehr vor. ${ }^{16}$

Der Ausdruck «Transzendenz» erscheint auch Laplanche merkwürdig, wie er selbstkritisch meint (vgl. Laplanche, 1987). Trotzdem bleibt er dabei und unterscheidet die zwei Begriffe «Transzendenz» und «Übertragung». Meinerseits verstehe ich unter Transzendenz eine Raum- und Zeitlosigkeit, also etwas, das unsere Vorstellungskraft übersteigt. Laplanche (Laplanche, 1987) peilt aber etwas ganz Bestimmtes damit an:

Der Elternteil ist für das Kind eher einer, dem Bedeutung denn Wissen zugeschrieben wird. Es gibt eine ursprüngliche Übertragung in der Kindheit - auch wenn diese in das Nebenprodukt Sexualität mündet - und es gibt die analytische Übertragung, welche nicht als eine Reproduktion, sondern als eine Wiederaufnahme dieses Prozesses der ursprünglichen Übertragung zu verstehen wäre. Es ist im Wesentlichen das, worauf ich anspiele, wenn ich von der «Übertragung der Übertragung» [innerhalb der Kur, resp. des Tro- 
ges; Anm.d.A.] und der Transzendenz der Übertragung [ausserhalb des Troges; Anm.d.A.] spreche. (S. 309, Hervorh. i. O.)

Ich begreife die Transzendenz im Laplanche'schen Sinne als Transzendenz der erwachsenen sexuellen Welt, die man als Kind nicht versteht und als Erwachsene gleichfalls nicht. Und wie Laplanche in seinem Vorwort zur Transzendenz in der Übertragung sagt: "genau diese Transzendenz selbst wird ausserhalb der Kur übertragen» (Laplanche, 1987, S. 6). Das heisst, es gibt auch nach absolvierter Analyse etwas ganz Grundsätzliches, das nicht verstanden wird - nämlich die eigene Fremdheit und jene des Anderen.

Oder um wieder auf den bekannten Alteritätsbegriff zurückzukommen, dass

ganz einfach der Andere anders ist, jedoch mit diesem Paradox oder dieser Doppelsinnigkeit: Er ist anders als ich, da er anders als er selbst ist. Die äussere Alterität verweist auf die innere Alterität. (Laplanche, 1996, S. 184, Hervorh.i. O.)

Anders ausgedrückt: Das Fremde im Anderen weist auf das eigene Fremde hin, das ängstigt, das nicht verstanden wird und deshalb nach aussen übertragen wird.

\section{Literatur}

Aichhorn, T. (2019). Freud arbeiten lassen. Die Dynamisierung der Sexualtheorie durch Jean Laplanche. Brandes \& Apsel.

Bénabou, M., Cornaz, L., Pélissier, Y., \& Liège, D. De. (2002). 789 néologismes de Jacques Lacan. EPEL.

Bourguignon, A., Cotet, P., Laplanche, J., \& Robert, F. (1989). Traduire Freud. PUF. Flaake, K. (1995). Zwischen Idealisierung und Entwertung - Probleme der Perspektiven theoretischer Analysen zu weiblicher Homo- und Heterosexualität. Psyche, 49(9-10), 867-885.

Fink, B. (2005). Eine klinische Einführung in die Lacansche Psychoanalyse-Theorie und Technik. Turia + Kant.

Freud, S. (1920g). Jenseits des Lustprinzips. GW XIII, S. 1-69.

Freud, S. (1937d). Konstruktionen in der Analyse. GW XVI, S. 114-155.

Knigge, A. Freiherr von (2006 [1796]). Über den Umgang mit Menschen. Reclam.

Koellreuter, A. (2001). Das Tabu des Begehrens. Zur Verflüchtigung des Sexuellen in Theorie und Praxis der feministischen Psychoanalyse. Psychosozial Verlag. 
Koellreuter, A. (Hrsg.). (2004). Faire travailler Freud. Festschrift zum 80. Geburtstag von Jean Laplanche. Werkblatt, 52(1).

Mathey, E. (2004). Gespräch mit Nadine und Jean Laplanche. In Koellreuter, A. (Hrsg.): Faire travailler Freud. Festschrift zum 80. Geburtstag von Jean Laplanche. Werkblatt, 52(1), 11-32.

Laplanche, J. (1987). Problématiques V. Le baquet-transcendance de transfer. PUF.

Laplanche, J. (1994). Nouveaux fondements pour la psychanalyse. PUF.

Laplanche, J. (1996). Die unvollendete kopernikanische Revolution in der Psychoanalyse. Fischer.

Laplanche, J. (1998). Die Psychoanalyse als Anti-Hermeneutik. Psyche, 52(7), $605-618$.

Laplanche, J. (2011). Neue Grundlagen für die Psychoanalyse. Die Urverführung, aus dem Französischen von H.-D. Gondek, herausgegeben von U. Hock \& J.-D. Sauvant. Psychosozial Verlag.

Macalpine, I. (1972). L'évolution du transfert. Revue Française de Psychanalyse, 3, $446-474$.

Passett, P. (2007). Unveröffentlichtes Manuskript zum Wartegg-Seminar «Das Denken von Jean Laplanche».

Sigusch, V. (1984). Vom Trieb und von der Liebe. Campus Verlag.

\section{Anmerkungen}

1 Zu finden in: Laplanche, J. (1987). Problématiques V. Le baquet-transcendance de transfert. PUF.

2 Der vorliegende Text ist eine überarbeitete Fassung des Vortrages, gehalten 2011 an der Tagung «Freud-Laplanche» der WPV und WPA in Wien.

3 Zufällig war dies der letzte Tag unseres zweijährlichen Laplanche-Treffens, vom 7.-9. Juli 2010 im Burgund.

4 Das grosse Projekt der Freud-Übersetzung begann Laplanche 1988 zusammen mit Kollegen, es ist noch nicht beendet. Im Text «Traduire Freud» (1989) wird die Arbeitsweise der Autoren beschrieben.

5 Von Bénabou und Kollegen sind 2002 aus Lacans gesamtem Werk «789 néologismes de Jacques Lacan» zusammengetragen worden.

6 Mit animalisch ist menschlich oder «lebewesenmässig» gemeint. Mesmer selbst sprach von tierischem Magnetismus.

7 Siehe auch http://www.noack-hypnose.de/hypnose-lexikon-franz-anton-mesmer. htm [8.6.2020].

8 Was unter eine Verschwörungstheorie zu subsumieren wäre, nach welcher Wissenschaftler monströse Maschinen verwendeten, um Gedanken zu steuern.

$9 \quad$ Alle weiteren Zitatübersetzungen aus Problématiques Vstammen von mir.

10 Siehe auch die Definition bei Wikipedia zu Potentialdifferenz [20.6.2020].

11 Ich verweise auf "sujet supposé savoir», ein von Lacan übernommener Begriff. 
12 In Bions container nimmt - kurz gefasst - die Analytikerin das Unerträgliche der Analysandin auf, formt es um in Erträgliches und gibt es in einem weiteren Schritt der Analysandin zurück.

13 Die Übertragungsdiskussion von Laplanche mit Macalpine und Lagache wäre ein eigenes Thema für sich.

14 Vgl. die von Hock und Sauvant herausgegebene deutsche Übersetzung von Laplanche (1994) zu Laplanche (2011, S. 197) oder auch Aichhorn (2019, S. 130). Letzterer übersetzte den Titel «le baquet - transcendance du transfer» in «Der Trog, die Übertragung der Übertragung» (S. 130), was meiner Meinung nach nicht gleichbedeutend mit Laplanches Transzendenz ist.

15 Dies entstammt einer persönlichen Mitteilung von Christophe Dejours, Präsident des Wissenschaftsrates der Fondation Laplanche und durch seine enge Zusammenarbeit mit Laplanche seit Jahrzehnten fundierter Laplanche-Kenner.

16 Zu vermuten ist, dass Laplanche sich nach seiner Auseinandersetzung mit Lacan in den 1970er Jahren zunehmend von diesem distanzierte und inhaltlich eigene Wege ging. «Leben und Tod in der Psychoanalyse» erschien 1970 in Paris und 1985 erstmals in deutscher Sprache mit dem Vermerk: «Ein Buch aus der Lacan-Schule im Walter-Verlag».

\section{Angaben zur Autorin}

Anna Koellreuter, Dr. phil., Psychoanalytikerin in eigener Praxis in Zürich, Mitglied Psychoanalytisches Seminar Zürich, bis 2019 in der Redaktion «Werkblatt. Zeitschrift für Gesellschaft und Psychoanalyse». Publikationen zur Analytikerin im Analyseprozess und zur Triebdynamik in der Analyse, u. a. erschienen im Psychosozial Verlag «Das Tabu des Begehrens. Zur Verflüchtigung des Sexuellen in Theorie und Praxis der feministischen Psychoanalyse» (2000) und «Wie benimmt sich der Prof. Freud eigentlich? Ein neu entdecktes Tagebuch von 1921 historisch und analytisch kommentiert» (2010). 